\section{A RADIOGRAPHIC STUDY OF THE MASTOID.}

\author{
By Ohiver A. Lothrop, M.D., Boston,
}

Assistant Otologist, Massachusetts General Hospital; Clinical Assistant, Massachusetts Charitable Eye and Ear Infirmray.

Trre following observations were made from a large series of $\mathrm{X}$-ray plates in possession of the Massachusetts Charitable Eye and Ear Infirmary and I wish to thank the various members of the staff for their permission and assistance. In many instances the X-ray interpretations could be veritied hy operative findings.

The use of the X-ray plate for aid in diagnosis of mastoid conditions is recent and promises to be of: great value, especially in differential diagnosis between an acute mastoiditis and an acute exacerbation of a chronic sclerosed mastoid. As a rule, the mastoid picture shows a more minute change in pathology than is shown in that of the accessory nasal sinuses. The reverse is true perhaps of transillumination. For detecting changes in the antrum of llighmore, for example, the transilluminator is more aceurate than the $\mathrm{X}$-ray, but in the mastoid the $\mathrm{X}$-ray will differentiate between a congested though cellular mastoid, one whose cells are broken down and one that is sclerosed, while the transillumination of the mastoid simply shows some pathologien condition obstructing to different degrees the rays of light.

T.t is not necessary to go into the details of how the $\mathrm{X}$-ray exposures are made and the plates developed. Suffice it to say that the side of the head to be skiagraphed is laid on the photographic plate with the film side up. Sometimes the auricle is left in its natural position and at other times it is turned forward. The rays are directed through the head from a point posterior to the opposite mastoid, so as to avoid superimposing any dense structures of the head. For this reason the bony external auditory canal is not shown as a distinct oval but more as a mottled aren, and other relations are more or less distorted. The more carefully a plate is exposed and developed, the more accurate and easy will be the interpretation. A sclerosed or normal cellular mastoid can be made out in almost the poorest plate but for interpretation of slight l,athological changes in acute matoiditis a good phate is an absolute necessity. Plates of both mastoids should be taken in all cases for comparison.

The normal cellular mastoid projection shows the cellular spaces clearly and the thin partitions are clear cut. Anterior to the cellular area is a roughly triangular, dense aren which is the petrous portion of the temporal bone. Within this area the bony meatus shows as a less dense and mottled spot. Had the projection been taken in the direction of the auditory canal, the meatus would have been shown as a hole. Anterior to this triangular area is seen the ascending ramus of the jaw and its articulation. 'The upper border of the triangular area is formed by the zygoma and its projection posteriorly as the linea temporalis. The middle cranial fossa lies above the border. The zygomatic cells, when present, often show projected in and above this border. The antrum in a normal, cellular mastoid is rarely distinguishable from the other cellular spaces. The position of the lateral sinus and sigmoid curve ean generally be made out but is not as distinct as in a selerosed mastoid. As in the latter, the anterior border is more distinct. In a general way, it passes from the upper, posterior part obliquely downward and forward to the bulb which lies under the middle ear. The tip is always seen as a distinet free horder. The tip may be more or less solid or filled with cells so as to leave only a shell-like cortex.

Posterior to the simus is seen the posterior cranial fossa in which lies the cerebellum. The suture lines of the occipital, parietal and mastoid bones often' ean be seen distinetly. In the young the cellular area is much restricted and the cells are generally smaller than in older individuals. Not unfrequently there is much variation in the size, shape and arrangement of cells in different cases but there is a striking similarity between the two mastoids of the same individual. If there is a small mastoid on one side, the other will be small. If one mastoid has small diploëtic cells, the other has also, or if one has large pneumatic cells, such are found in the other. The outline of the cellular area is generally similar in both. The auricle must be mentioned here, not because we desire its projection, but because its shadow frequently masks or accentuates some border and allowance must be made for it.

The $X$-ray picture of the mastoid in acute conditions varies as the pathological processes advance. In the majority of cases of acute otitis media the mastoid cells are affected simultaneously, even though the pathological condition be only a participating congestion. In all severe infections the mastoid cells are probably likewise infected, and pus from the mastoid is added to that from the middle ear. In this congestive stage the natural cellular appearance of the mastoid would be preserved with the exception that there would be a loss of definition between the cell spaces and their partitions. In fact the whole plate of the mastoid region shows less penetration to light than the other side. This is the most diffeult stage of mastoid inflammation to interpret. Almost perfect plates of both sides, equally exposed and developed, are required. This picture can be simulated by other inflammations and congestions of the surrounding soft parts, most commonly, furunculosis of the external auditory canal. Fortunately, this 
stage of mastoid involvement rarely requires surgical intervention.

After six to ten days the picture changes in cases of virulent mastoiditis. There is now seen a distinct blurring of the mastoid structure. The cell partitions are barely made out and there is very little contrast between the cell spaces and their partitions. At the end of this time, actual loss of some cell partitions may be made out. In such a mastoid, the cell spaces would be found filled with pus, the mucous membrane lining swollen and polypoid, and there would be some softening of the bony partitions.

Plates of mastoiditis which has progressed for two, three or four weeks show still different pictures and usually are easy to interpret. At this stage is noted distinctly loss of cell partitions, especially in the tip or in the center over the lateral sinus. Areas less affected by the pathological changes still show blurred cellular structure. Later, well advanced cases may show no cellular structure in any part and may even show cavity formation due to destruction of bone. These mastoids, as found at operation, are very vascu. lar, broken down, and filled with granulations and a little pus.

Perisinous abscess in the central portion of the mastoid is sometimes diagnosed in those cases above spolken of. where cavity formation is noticed is that region. Where the bony wall of the sinus and the cellular structure over it has been absorbed by the pathological process, it is conceivable that such a cavity should show in the plate because the rays can thus more easily penetrate that area. A perisinous abscess well posterior, and an extradural abscess, both being partially beyond the limits of the normal cellular mastoid, frequently show up distinctly. The impression is that of a cavity or less dense area surrounded by a dense areola fading off gradually above and posteriorly. A brain abscess has not been observed. Sinus thrombosis is probably not recognizable to any extent. There is no reason to believe that clotted blood has any different effect on the penetration of the rays than fluid blood. The reason for the impression of the lateral sinus on the plate will be spoken of in connection with the sclerosed mastoid.

There are many cases of mastoiditis that never come to operation and, after weeks or months, the majority get well. The X-ray seems to throw some light on what happens to the mastoid in these cases and how nature cures them. It has been mentioned that the cell partitions are absorbed as the process goes on and all the spaces are filled with granulations and the quantity of pus is diminished as the virulence of the infection is gradually overcome. Have we not now a condition similar to that after operation when the mastoid cavity has been cleaned out and later filled with granulations in the process of healing? What happens to the post-operative cavity? Regenerated bone, usually homogeneous. but rarely cellular, fills the cavity as is seen in
X-ray plates of old post-operative cases and as is found in operating on cases of recurrent mastoiditis. In the post-operative cases the bone regenerates from the periphery of the cavity and frequently leaves a seam of fibrous tissue directly under the cicatrix of the incision. It is also observed that bone is not regenerated in the area where the sinus was uncovered by operation. With the above clinical and $\mathrm{X}$-ray evidence that post-operative mastoid cavities fill in with granulations followed by regenerated bone, can it be doubted that this same process takes place in an unoperated case cured by nature? A few of these transition cases were found in which the $\mathrm{X}$-ray showed almost a homogeneous shadow and at operation a soft, bleeding, regenerating bone and granulation tissue was found but no pus. If for any reason, as, for example, carious ossicles on a marginal perforation, the antrum and middle ear did not heal as the mastoid was filling in with bone, a chronic suppurative ear would result.

We have considered above what takes place in an adult, fully developed mastoid. Let us now consider what would be the result in babies and young children where there is only an antrum and rarely a few cells budding off. In this group occur a goodly proportion of our acute suppurating ears and it is a well known fact that discharge from a baby's ear is very chronic. When operation is performed the antrum is found filled with granulations. Though the antrum is of about alult size, the mastoid tip and surrounding parts are proportionally smaller and close to one another. The normal antral lining has been destroyed so that as the child grows the budding off of cells will not take place. The bony parts will enlarge some but not in proportion to those filling with cells. The sinus is left nearer the antrum and the groove is deeped in the bony parts. Does not this explain the small, sclerosed mastoid of later life with its ivory like texture and its lateral sinus so close to the posterior wall that easy access to the antrum is impossible? On the other hand, how rarely does a lateral sinus obstruct the surgeon's passage to the antrum in an acute cellular mastoid.

Let us now consider the appearance of the sclerosed mastoid in the X-ray plate. The sclerosed mastoid is the type always seen in cases that have or have had a chronic suppurative middle ear and mastoid. So constant is this relation that with one exception, all cases diagnosed clinically as a chronic suppurative ear showed a sclerosed mastoid. The one exception was a case of atresia of the canal with granulations and foul pus. The mastoid was cellular and the middle ear normal after curing the canal. The reverse, however, is not so constant. Acute exacerbations of a chronic suppurative ear or acute otitis media in an ear which was once a chronic suppurative ear frequently can- 
not be differentiated clinically from an acute attack in an otherwise normal ear. Here, the $\mathrm{X}$-ray shows us the past history as represented by a sclerosed mastoid. And again, given an X-ray plate of a sclerosed mastoid, it is practically impossible to detect the presence of an acute inflammation.

In a completely sclerosed mastoid no cells whatever can be seen. The bone looks smooth and dense. The meatus and middle ear are seen as in normal mastoids as a less dense area. Usually the antrum shows as a mottled area just above and posterior to the meatus. The lateral sinus generally shows very distinctly in this type of mastoid. The more dense the sclerosed bone and the longer it has existed, the more distinctly does the sinus stand out. The anterior border of the sinus is generally more distinct than the posterior. In some cases the latter can hardly be defined from the surrounding bone. The sinus shows so clearly in this type of mastoid because of the fact that the sigmoid groove is very deep in the mastoid bone. This makes it easy for the rays to pass through the sinall amount of bony cortex over the path of the sinus while it is difficult to penetrate the mass of selerosed bone on the borders. The fact that there is llood in the sinus does not help to make it conspicuous. On the contrary, it would tend to limit the contrast because light can pass less ensily through blood. In cases of cellular mastoids, the dense borders are replaced by cells which obstruct the rays very little and this explains the inconspicuousness of the sinus in the normal mastoid. The position of the sinus varies in the different cases and the position in the plate is of some value to the surgeon before operating. Onc cannot lay a measure on the plate and say that the sinus is just so many millimeters posterior to the posterior wall of the canal. The projection so measured would be deceptive for several reasons. First, the Röntgenologist may have placed the heads at slightly different angles, or the shape of the individual head made this imperative. The course of the sinus not only varies, as seen in a lateral projection, but may be nearer the surface in some cases than in others. The more marked the light penetration over the sinus, the more superficial it must be. The more acute the angle at the knee (junction between sinus proper and sigmoid portion), and at the same time superficial and projected close to the posterior wall, the more troublesome it will prove while excavating a passage to the antrum. In some cases the anterior wall of the sinus can be seen projected over the posterior and inferior portion of the antrum and in such cases the sinus is liable to be uncovered before reaching the antrum.

Some sclerosed mastoids, especially the larger ones, occasionally show a large area about the antrum or extending down toward the center which is less dense and mottled in appearance. At operation such a mastoid is found to be brok- en down with areas of pus, granulations of cholesteatome. Sometimes a distinct cholesteatome cavity may be seen.

'The appearance of the tip of a sclerosed mastoid which dates from infancy is often characteristic. As had been said, the tip cannot be developed by budding cells from the antrum nor can it be filled in by regenerated bone after destruction of the existing cells as in cases in which the sclerosing process started later in life. In these cases the tip is densely sclerosed but has fine wavy lines, as is seen in ivory. 'This appearance has been interpreted as diploëtic but is not found so at operation. These cases give the best detailed pictures and at times the mastoid vein and foramen is seen and on two occasions the Fallopian canal could be made out. A line of fracture through the mastoid was shown clearly in a plate and later was demonstrated by operation.

There is one type of case that has not yet been spoken of, namely, the so-called effectus ear. By that term is meant any ear which shows hy scars or perforation of the drum membrane that there has been a past suppuration. An $X$-ray plate of such an ear shows all degrees, from a normal cellular mastoid, partly cellular, semi-sclerosed to a completely sclerosed mastoid. the same patient may have a sclerosed mastoid, on one side and a semi-sclerosed or normal mastoid on the other and yet both ear drums are effectus and there is history of past ear trouble. This observation may be easily explained. It is well known that a mild acute ear can cause a scar by rupture of the drum or by having a paracentesis done, suppurate for a short while and then dry up without distinctive involvement of the mastoid and yet be classified as an effectus ear. Iad this process gone farther before clearing up there would have been more damage to the drum and the mastoid would have been structurally altered. Partial sclerosis, which always advances from the periphery, would have resulted. The cells remaining were either uninjured cells that escaped the suppuration or a regeneration of cells, the latter having been observed clinically by others. The fact has been known clinically that a discharge existing for years in a chronic suppurative ear may cease and the drum cicatrize and in these cases is to be found the explanation of the sclerosed mastoid seen in an effectus ear. Nothing shows more clearly than X-rays of effectus ears the varying degrees in which suppurations in the middle ear affect the mastoid structure.

The following more detailed statisties may be of interest. The total number of mastoid pictures tabulated was 460 . Many others were discarded either because the plates were poor or because of insufficient data. The number of pathological mastoids was about evenly divided between the right and left sides. There were many more sclerosed mastoids on the right side 
than on the left but the number of acute mas- cellular or normal. In cases where no diagnotoids on the left side equalized the division. This coincides with the records of the mastoid operations performed at the Infirmary for the past ten years. Although one side may prevail over the other for a given period, the average is about the same for each side.

In cases where the clinical diagnosis of chronic suppurative ear was made, $94 \%$ showed total sclerosis of the mastoid and $6 \%$ semi-sclerosis. In the cases where a diagnosis of effectus ear was made, $56 \%$ were totally sclerosed, $27 \%$ semi-sclerosed, and $17 \%$ were five cases.

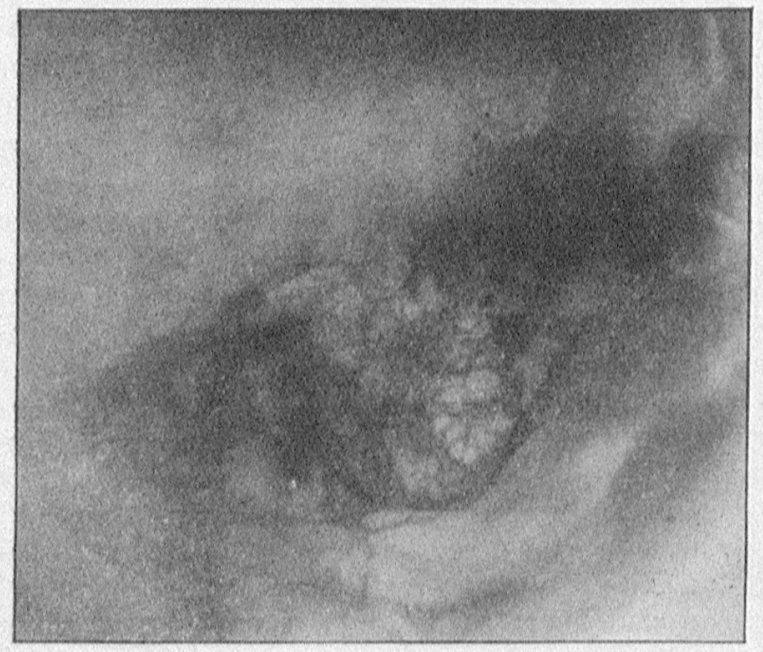
sis of a past or present suppurative process was made, $92 \%$ had normal mastoids and $8 \%$ were sclerosed or semi-sclerosed. $10 \%$ of the cases diagnosed as acute suppurative otitis media showed sclerosed or semi-sclerosed mastoids. These represent the cases of acute processes superimposed on an effectus ear, or the acute exacerbation of a chronic ear. These latter are often difficult to differentiate clinically. The conditions found at operation were compared with the clinical and $x$-ray diagnoses in seventy-

A left normal cellular. mastoid. Female. Age 26. The right mastoid was sc'lerosed from a chronic suppurative ear of 12 years' duration.

M C, mastoid cells. Z C, zygomatic cells. Z, zygoma. ME, mirlclle ear and cunal. $j$, ascending ramus of lower jaw. $T$, mastoid tip.
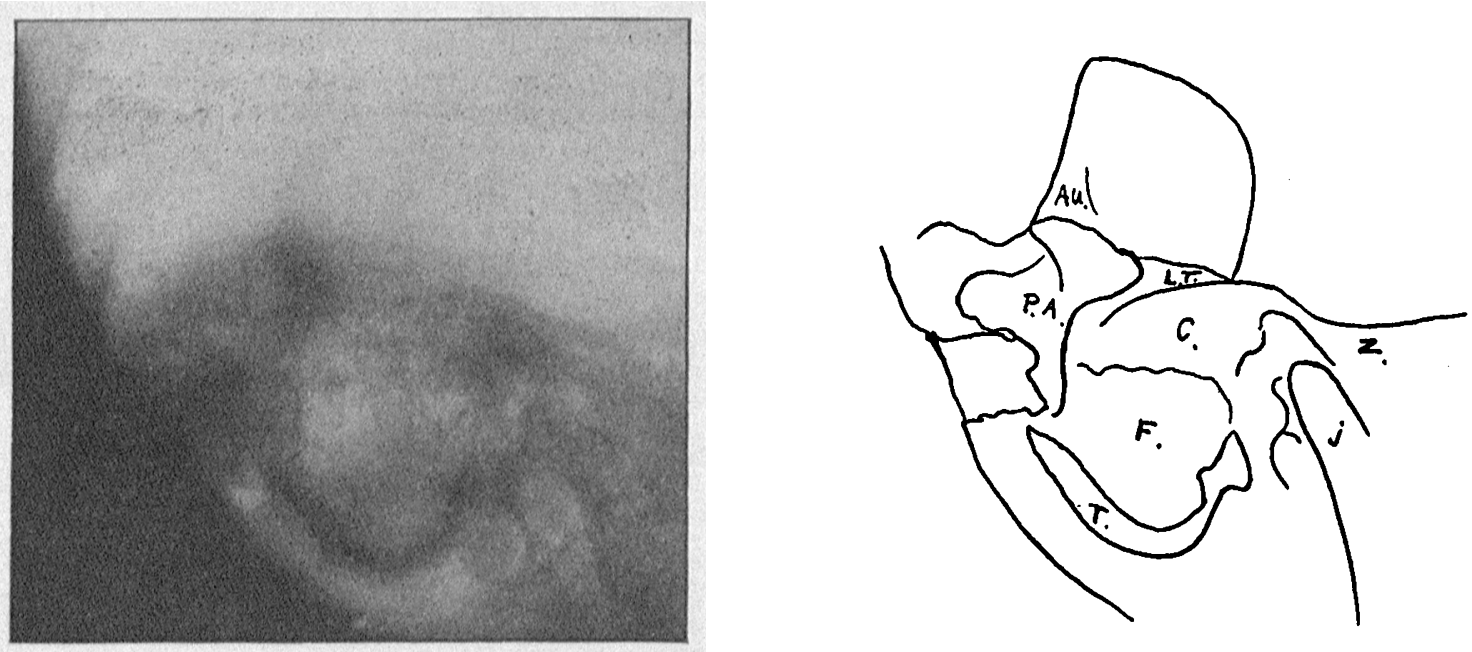

$\Lambda$ right acute destructive mastoiditis, Male. Age $48 . \quad$ A discharging ear for two morths.

$\mathrm{T}$, remnins of mastoid cortex at tip. $\mathrm{F}$, homogeneous area where all cellular structure is replaced by pus and granulations. $C$, mottled aren Huggesting some remnants of cellular structure bounded above by the linea temporalis. P A, shadow due to postunrul absecss and gramulations over the sinus. $j$, ascending ramus of lower jaw. Au, auricle. At operation there was found r iniral abscess, perforation of the cortex and the lound a postwith pus and rranulatious. Some cellular structure was found ubout the untrun. 

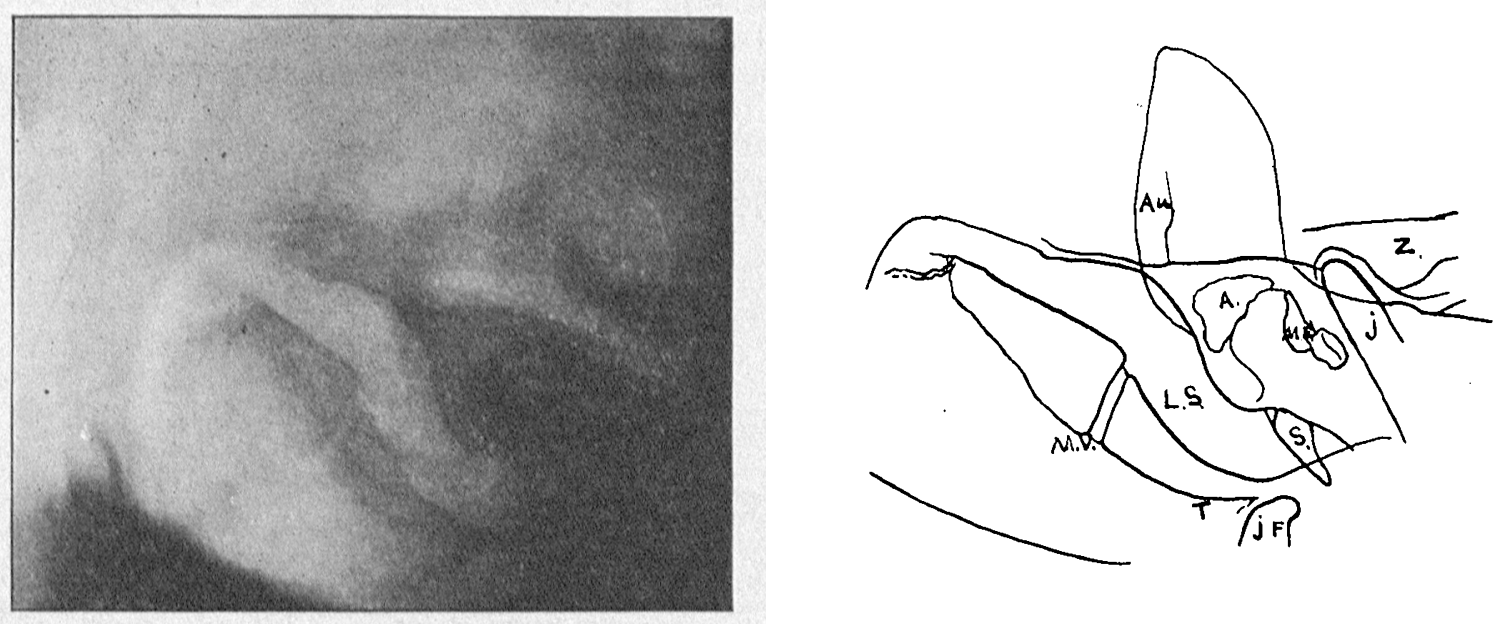

A right sclerosed mastoid. Female. Age 12. The left mastoid was similarly sclerosed and verified at operation. Both ears had discharged since five years of age.

$\mathrm{Au}$, auricle. L S, lateral sinus. S, styloid process. Z, zygoma. J, ascending ramus of lower jaw with the articulation. It $\mathrm{E}$, milddle ear. $M V$, mustoid foramen and vein. $T$, sclerosed tip. jF, jugular formunen. $A$, less dense area in sclerosed bone suggesting sllic at alt trill1.
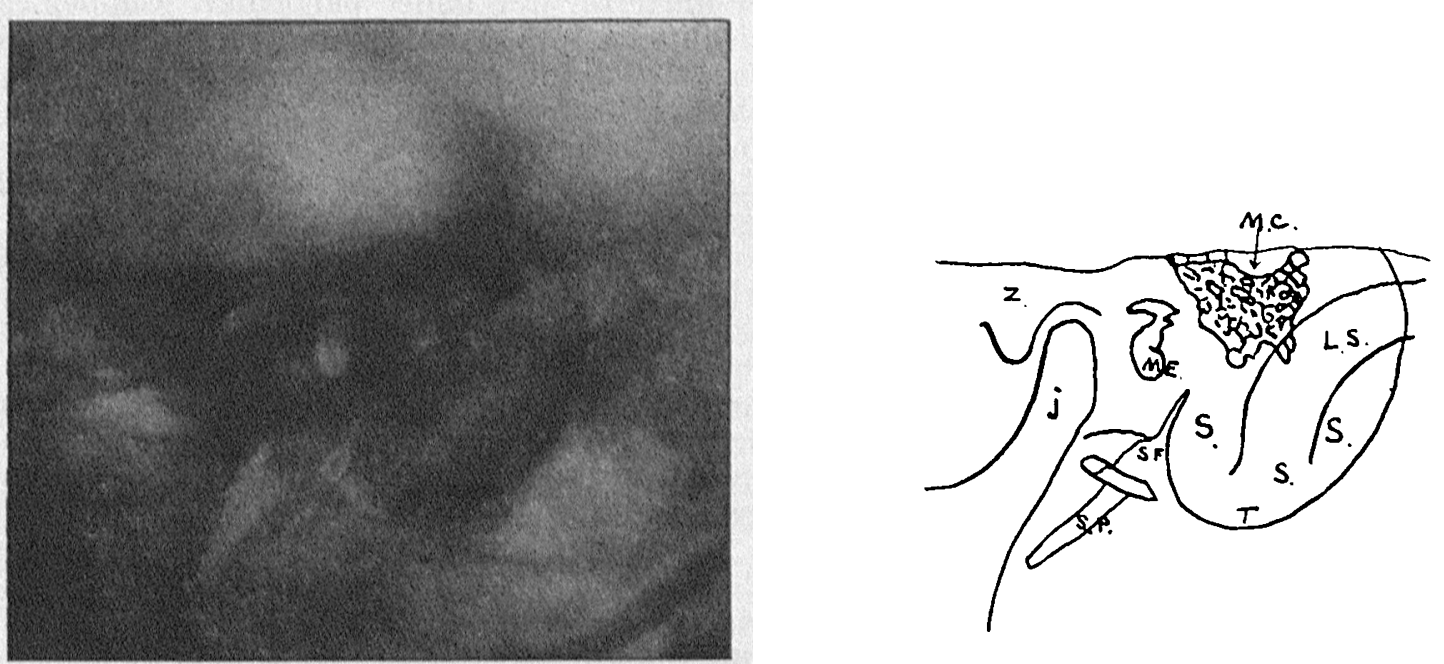

A left semi-sclerased mustoid. Male. Age 50.

sf $\mathrm{E}$, middle ear and canal and shadow of maleus. Z, zygoma. $j$, ascending ramus of lower jaw. M $\mathrm{O}$, remnants of some ill-deflned mastoid cells L S, Interal sinus. Posterior border not distinct. $\mathrm{S} S \mathrm{~S}$, sclerosed area. The wavy ivory-like structure is visible in the negative. T, mastoid tip. S P, styloid process $\mathrm{S} F$, stylo. mastoid foramen and Fallopian canal. 

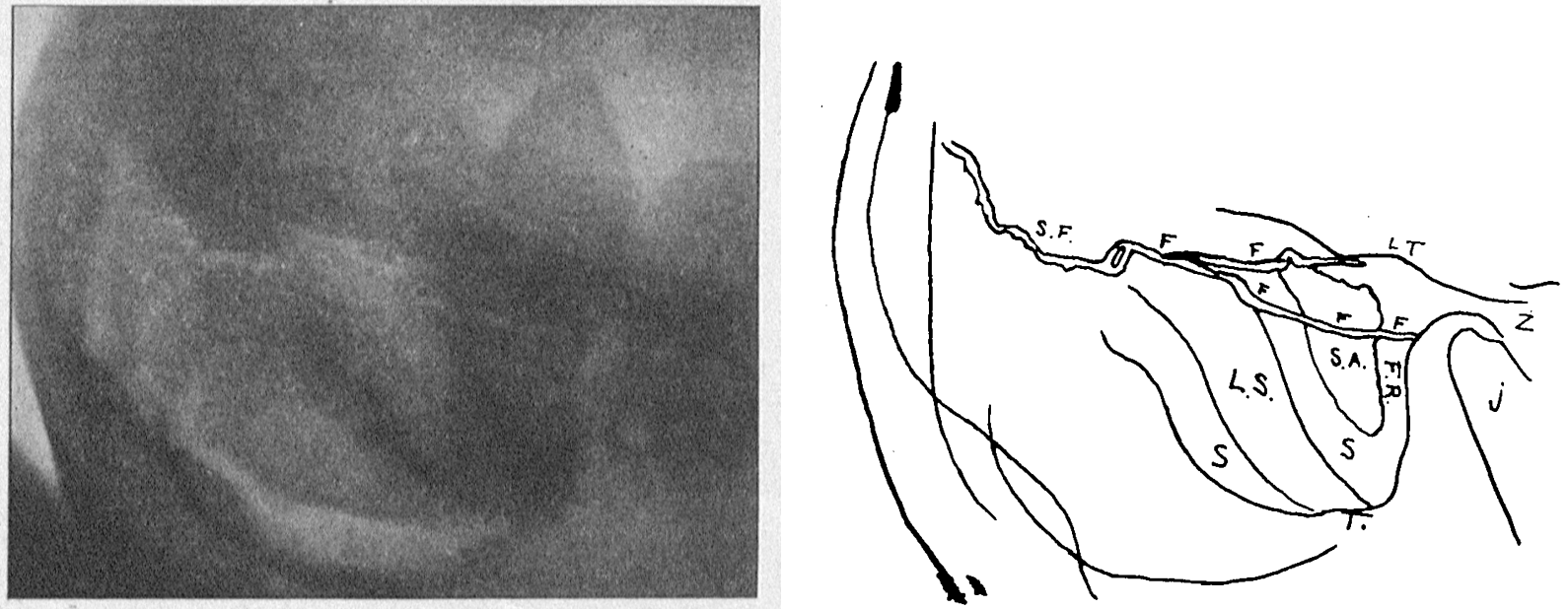

A right sclerosed mastoid showing a line of fracture. Male. Age 36. The left ear was an effectus ear and the mastoid sclerose. The richt patient had a right facial paralysis for that length of time

patient had a right facial paralysis for that length of time. F F F, line of fracture from the occipital suture (S F) across the mastoid and facial ridge (FR). $\mathrm{S} A$, softened area in the mastoid. $8 \mathrm{~S}$, sclerosed arca. $T$, mastoid tip. LS, lateral sinus. L $T$, linea temporalia $Z$, zygoma. $j$, ascending ramus of jaw

At operation the line of fracture was fou

\section{LABORATORY PHYSIOLOGY IN COLLEGE AND IN MEDICAL SCHOOL.}

By Artiun Dermont Bush, M. D., Bundngton, Vt

Instructor in l'hysiology, Univ. of Vermont, College of Medicine.

In 1885, Professor John C. Draper of the Medical Department of the University of the City of New York published his highly interesting book entitled "Medical Physics." By way of introduction he says: "It is no exaggeration to say that there is not a tissue, organ or function of the body, the proper comprehension of which does not involve a knowledge of the laws of physies. We may with equal justice affirm that there is scarcely a pirinciple of physics which is not applied in some form in the human body." He then laments the general ignorance of physics by American physicians, especially as compared with Europeans, and offers his text as a wholesome corrective. $\mathrm{He}$ deals, somewhat in extenso, with fundamental principles, but makes many pioneer applications of these principles to the science of medicine.

Nearly thirty years have passed since this work of Dr. Draper first appeared. During this period the teaching of Physics as a separate subject has been taken up by many schools, carried for a while, and subsequently dropped; not, however, without distinctly affecting methods of teaching. We take note of physical principles in pathology, surgery and hygiene, but more particularly in physiology a greater part of whose manifestations are simply phy- sical. Yet in these several branches of medicine, use is made chiefly of such of the data of physics as are directly applicable; the broad general principles are no longer taught in the medical school. We have come to see that medical science is specific, not general; deals not with the universality of law, but with the operating of universal law under particular, well-defined conditions. As a consequence of this change in our view-point a knowledge of the science of physics is now demanded not as a part of the medical course but as a necessary prerequisite. Knowledge of physical laws is, as Draper urged, essential to the proper comprehension of bodily functions, but that knowledge must be acquired where universals are taught, - in the academic side of the university; whereas a knowledge of the specific applications of those laws to the functioning human body must be learned in that department of the university devoted to the special study of the human body, to wit, in the medical school.

A similar comparison might be drawn from the methods of teaching chemistry. Not so many years ago, nearly all schools used valuable time teaching medical students fundamental principles in chemistry:- the laws of chemical combination and dissociation, nomenclature, periodicity, atomic theory, reactions and compounds of the metals and non-metals. Today, however, all of this general knowledge must be acquired before entering the medical school; and we are looking forward to the time when organic chemistry together with qualitative and quantitative analyses will also be a part of all premedical courses. Here again we recognize the principle that knowledge of general application should 